\title{
Iterative Multistep Reproducing Kernel Hilbert Space Method for Solving Strongly Nonlinear Oscillators
}

\author{
Banan Maayah, ${ }^{1}$ Samia Bushnaq, ${ }^{2}$ Shaher Momani, ${ }^{1,3}$ and Omar Abu Arqub ${ }^{4}$ \\ ${ }^{1}$ Department of Mathematics, Faculty of Science, The University of Jordan, Amman 11942, Jordan \\ ${ }^{2}$ Department of Science, Faculty of Science, Princess Sumaya University for Technology, Amman 11941, Jordan \\ ${ }^{3}$ Nonlinear Analysis and Applied Mathematics (NAAM) Research Group, Faculty of Science, King Abdulaziz University, \\ Jeddah 21589, Saudi Arabia \\ ${ }^{4}$ Department of Mathematics, Faculty of Science, Al Balqa Applied University, Salt 19117, Jordan \\ Correspondence should be addressed to Shaher Momani; s.momani@ju.edu.jo
}

Received 26 March 2014; Revised 21 May 2014; Accepted 22 May 2014; Published 17 June 2014

Academic Editor: Shao-Ming Fei

Copyright (C) 2014 Banan Maayah et al. This is an open access article distributed under the Creative Commons Attribution License, which permits unrestricted use, distribution, and reproduction in any medium, provided the original work is properly cited.

A new algorithm called multistep reproducing kernel Hilbert space method is represented to solve nonlinear oscillator's models. The proposed scheme is a modification of the reproducing kernel Hilbert space method, which will increase the intervals of convergence for the series solution. The numerical results demonstrate the validity and the applicability of the new technique. A very good agreement was found between the results obtained using the presented algorithm and the Runge-Kutta method, which shows that the multistep reproducing kernel Hilbert space method is very efficient and convenient for solving nonlinear oscillator's models.

\section{Introduction}

Nonlinear oscillators have several applications in many fields of physics, engineering, and biology [1-4]. In general, nonlinear oscillator's problems are sometimes too complicated to be solved exactly, so several numerical methods are proposed by many authors such as harmonic balance method, multiple scale method, Adomian decomposition method, homotopy perturbation method, homotopy analysis method, and differential transform method. The reader is kindly requested to go through [5-17] in order to know more details about these methods, including their history, their kinds and types, their modification for use, their scientific applications, and their characteristics.

Reproducing kernel theory has important applications in numerical analysis, differential equations, integral equations, integrodifferential equations, and probability and statistics [18-20]. Recently, a lot of research work has been devoted to the applications of RKHS method for wide classes of stochastic and deterministic problems involving operator equations, differential equations, integral equations, and integrodifferential equations. The RKHS method was successfully used by many authors to investigate several scientific applications side by side with their theories. The reader is kindly requested to go through [21-32] in order to know more details about RKHS method, including its history, its modification for use, its scientific applications, its kernel functions, and its characteristics.

The new algorithm is a simple modification of the RKHS method, for finding approximate solutions to the linear and nonlinear oscillator's equations in large intervals. It is found that the corresponding RKHS method is valid only for short intervals, but, by using multistep RKHS method, more valid and accurate solutions over large intervals can be obtained. The new method has the following characteristics; first, it is of global nature in terms of the solutions obtained as well as its ability to solve other mathematical, physical, and engineering problems; second, it is accurate, needs less effort to achieve the results, and is developed especially for the nonlinear case; third, in the proposed method, it is possible to pick any point in the interval of integration and as well the approximate solutions will be applicable; fourth, the method does not require discretization of the variables, and it is not affected by computation round-off errors and one 
is not faced with necessity of large computer memory and time; fifth, the proposed approach does not resort to more advanced mathematical tools; that is, the algorithm is simple to understand and implement and should be thus easily accepted in the mathematical and engineering application's fields.

This paper is comprised of four sections including the Introduction. In Section 2 we describe the multistep RKHS method. In Section 3 we present four examples to show the efficiency and simplicity of the method. The conclusions are given in Section 4.

\section{Multistep Reproducing Kernel Hilbert Space Method}

In functional analysis, the RKHS is a Hilbert space of functions in which pointwise evaluation is a continuous linear functional. Equivalently, they are spaces that can be defined by reproducing kernels. In this section, we utilize the reproducing kernel concept to construct two reproducing kernel Hilbert spaces and to find out their representation of reproducing functions for solving second-order oscillator equation via RKHS technique.

Let us consider the following second-order nonlinear oscillator equation:

$$
u^{\prime \prime}(x)=f\left(x, u(x), u^{\prime}(x)\right), \quad a \leq x \leq b,
$$

subject to the initial conditions $u(a)=\alpha, u^{\prime}(a)=\beta$.

Definition 1. Let $\mathscr{H}$ be a Hilbert space of function $f: \chi \rightarrow \mathscr{F}$ on a set $\chi$. A function $K: \chi \times \chi \rightarrow \mathbb{C}$ is a reproducing kernel of $\mathscr{H}$ if the following are satisfied. Firstly, $K(\cdot, x) \in \mathscr{H}$ for all $x \in \chi$. Secondly, $\langle f, K(\cdot, x)\rangle=f(x)$ for all $f \in \mathscr{H}$ for all $x \in \chi$.

Remark 2. The last condition in Definition 1, called "the reproducing property," means that the value of the function $f$ at the point $x$ is reproduced by the inner product of $f$ with $K(\cdot, x)$.

It is worth mentioning that the reproducing kernel $K$ of a Hilbert space $\mathscr{H}$ is unique, and the existence of $K$ is due to the Riesz representation theorem, where $K$ completely determines the space $\mathscr{H}$. Moreover, every sequence of functions $f_{1}, f_{2}, \ldots, f_{n}, \ldots$ which converges strongly to a function $f$ in $\mathscr{H}$ converges also in the pointwise sense. This convergence is uniform on every subset on $E$ on which $x \rightarrow$ $K(x, x)$ is bounded. In this occasion, these spaces have wide applications including complex analysis, harmonic analysis, quantum mechanics, statistics, and machine learning. Subsequently, the space $W_{2}^{3}[a, b]$ is constructed in which every function satisfies the initial conditions $u(a)=\alpha, u^{\prime}(b)=$ $\beta$ and then utilized the space $W_{2}^{1}[a, b]$. For the theoretical background of reproducing kernel Hilbert space theory and its applications, we refer the reader to [18-20].
Definition 3 (see [21]). A Hilbert space $\mathscr{H}$ of functions on a nonempty abstract set $E$ is called a reproducing kernel Hilbert space if there exists a reproducing kernel $K$ of $\mathscr{H}$.

Definition 4 (see [24]). The inner space $W_{2}^{3}[a, b]=\{u$ : $u^{(j)}(x)$ are absolutely continuous real-valued functions on $[a, b], u^{(j)}(x) \in L^{3}, j=0,1,2$, and $u(a)=0, u^{\prime}(b)=$ $0\}$, where the inner product and the norm in $W_{2}^{3}[a, b]$ are defined, respectively, by $\langle u(x), v(x)\rangle_{W_{2}^{3}}=\sum_{i=0}^{2} u^{(i)}(a) v^{(i)}(a)+$ $\int_{a}^{b} u^{(3)}(x) v^{(3)}(x) d x$ and $\|u\|_{W_{2}^{3}}=\sqrt{\langle u(x), u(x)\rangle_{W_{2}^{3}}}$ in which $u, v \in W_{2}^{3}[a, b]$.

The space $W_{2}^{3}[a, b]$ is a reproducing kernel if, for each fixed $x \in[a, b]$ and any $u(x) \in W_{2}^{3}[a, b]$, there exist $K_{x}(y) \in$ $W_{2}^{3}[a, b], y \in[a, b]$ such that $\left\langle u(y), K_{x}(y)\right\rangle_{W_{2}^{3}}=u(x)$.

Theorem 5 (see [23]). The space $W_{2}^{3}[a, b]$ is a reproducing kernel and its reproducing kernel function $K_{x}(y)$ can be written as

$$
K_{x}(y)= \begin{cases}k(x, y), & y \leq x \\ k(y, x), & y>x\end{cases}
$$

where $k(x, y)=(1 / 120)(a-y)^{2}\left(-6 a^{3}-5 x y^{2}+y^{3}+10 x^{2}(3+\right.$ $\left.y)+3 a^{2}(10+5 x+y)-2 a\left(5 x^{2}-y^{2}+5 x(6+y)\right)\right)$.

Definition 6 (see [21]). The inner product space $W_{2}^{1}[a, b]$ is defined as $W_{2}^{1}[a, b]=\{u: u(x)$ is absolutely continuous real-valued function on $\left.[a, b], u(x), u^{\prime}(x) \in L^{2}[a, b]\right\}$, where the inner product and the norm in $W_{2}^{1}[a, b]$ are defined, respectively, by $\langle u(x), v(x)\rangle=\int_{a}^{b} u(x) v(x)+u^{\prime}(x) v^{\prime}(x) d x$ and $\|u\|_{W_{2}^{1}}=\sqrt{\langle u(x), u(x)\rangle_{W_{2}^{1}}}$ in which $u, v \in W_{2}^{1}[a, b]$.

Theorem 7 (see [21]). The Hilbert space $W_{2}^{1}[a, b]$ is a complete reproducing kernel and its reproducing kernel function $R_{x}(y)$ can be written as

$$
\begin{aligned}
R_{x}(y)= & \frac{1}{2 \sinh (b-a)} \\
& \times[\cosh (x+y-b-a)+\cosh (|x+y|-b-a)] .
\end{aligned}
$$

Reproducing kernel functions possess some important properties such as being symmetric, unique, and nonnegative. The reader is asked to refer to [18-32] in order to know more details about reproducing kernel functions, including their mathematical and geometric properties, their types and kinds, and their applications and method of calculations.

In order to apply the proposed algorithm of multistep RKHS easily, we need to homogenize the initial conditions $u(a)=\alpha$ and $u^{\prime}(a)=\beta$. To do so, let $v(x)=u(x)-(\beta x+(\alpha-$ $a \beta)$ ); then, (1) can be formulated in new form as follows:

$$
v^{\prime \prime}(x)=F\left(x, v(x), v^{\prime}(x)\right),
$$

subject to the initial conditions $v(a)=0, v^{\prime}(a)=0$, where $F\left(x, v(x), v^{\prime}(x)\right)=f\left(x, v(x)+(\beta x+(\alpha-a \beta)), v^{\prime}(x)+\beta\right)$. 
Anyhow, define the operator $L: W_{2}^{3}[a, b] \rightarrow W_{2}^{1}[a, b]$ such that $L v(x)=v^{\prime \prime}(x)$. Hence, (4) can be converted into the equivalent form depending on (4) as

$$
L v(x)=F\left(x, v(x), v^{\prime}(x)\right), \quad a \leq x \leq b,
$$

where $x \in[a, b], v(x) \in W_{2}^{3}[a, b]$, and $F\left(x, v(x), v^{\prime}(x)\right) \in$ $W_{2}^{1}[a, b]$.

Now, we construct an orthogonal function system of the space $W_{2}^{3}[a, b]$. For a countable dense set $\left\{x_{i}\right\}_{i=1}^{\infty}$ of $[a, b]$, let $\varphi_{i}(x)=R_{x_{i}}(x)$ and $\psi_{i}(x)=L^{*} \varphi_{i}(x)$ where $R_{x_{i}}(x)$ is the reproducing kernel space of $W_{2}^{1}[a, b]$ and $L^{*}$ is the adjoint operator of $L$. The orthonormal system $\left\{\bar{\psi}_{i}(x)\right\}_{i=1}^{\infty}$ of $W_{2}^{3}[a, b]$ can be derived from the Gram-Schmidt orthogonalization process of $\left\{\psi_{i}(x)\right\}_{i=1}^{\infty}$ :

$$
\bar{\psi}_{i}(x)=\sum_{k=1}^{i} \beta_{i k} \psi_{k}(x), \quad i=1,2, \ldots,
$$

where $\beta_{i k}$ are orthogonalization coefficients given as $\beta_{11}=$ $1 /\left\|\psi_{1}\right\|, \beta_{i i}=1 / d_{i}$, and $\beta_{i j}=-\left(1 / d_{i}\right) \sum_{k=j}^{i-1} c_{i k} \beta_{k j}$ for $j<i$ in which $d_{i}=\sqrt{\left\|\psi_{1}\right\|^{2}-\sum_{k=1}^{i-1} c_{i k}^{2}}, c_{i k}=\left\langle\psi_{i}, \bar{\psi}_{i}\right\rangle_{W_{2}^{2}}$ and $\left\{\psi_{i}(x)\right\}_{i=1}^{\infty}$ are the orthonormal system in $W_{2}^{3}[a, b]$.

Theorem 8. If $\left\{x_{i}\right\}_{i=1}^{\infty}$ is dense on $[a, b]$, then $\left\{\psi_{i}(x)\right\}_{i=1}^{\infty}$ is the complete system of $W_{2}^{3}[a, b]$ and $\psi_{i}(x)=\left.L_{y} K_{x}(y)\right|_{y=x_{i}}$. The subscript $y$ by the operator $L$ indicates that the operator $L$ applies to the function of $y$.
Theorem 9. If $\left\{x_{i}\right\}_{i=1}^{\infty}$ is dense on $[a, b]$ and the solution is unique on $W_{2}^{3}[a, b]$, then the solution of (5) is given by

$$
v(x)=\sum_{i=1}^{\infty} \sum_{k=1}^{i} \beta_{i k} F\left(x_{k}, v\left(x_{k}\right), v^{\prime}\left(x_{k}\right)\right) \bar{\psi}_{i}(x),
$$

and the solution of (1) satisfies the form

$$
\begin{aligned}
u(x)= & \left(\sum_{i=1}^{\infty} \sum_{k=1}^{i} \beta_{i k} F\left(x_{k}, v\left(x_{k}\right), v^{\prime}\left(x_{k}\right)\right) \bar{\psi}_{i}(x)\right) \\
& +\beta x+(\alpha-a \beta) .
\end{aligned}
$$

Here, the approximate solution $u_{n}(x)$ can be obtained by taking finitely many terms in the series representation of $u(x)$ and $u_{n}(x)=\sum_{i=1}^{n} \sum_{k=1}^{i} \beta_{i k} F\left(x_{k}, v\left(x_{k}\right), v^{\prime}\left(x_{k}\right)\right) \bar{\psi}_{i}(x)+\beta x+$ $(\alpha-a \beta)$. Also, since $W_{2}^{3}[a, b]$ is a Hilbert space, it is clear that $\sum_{i=1}^{\infty} \sum_{k=1}^{\infty}\left(\beta_{i k} F\left(x_{k}, v\left(x_{k}\right), v^{\prime}\left(x_{k}\right)\right)\right)+\beta x+(\alpha-a \beta)<\infty$. Therefore, the sequence $u_{n}$ is convergent in the norm.

The major aim of this work is to find the approximate solution to (1). Next, we utilize the multistep RKHS procedure; to do so, we consider the nonlinear initial value problem (IVP) of (1). Indeed, let $[0, T]$ be the interval over which we want to find the solution of the IVP (1). Assume that the interval $[0, T]$ is divided into $M$ subintervals $\left[x^{m-1}, x^{m}\right], m=$ $1,2, \ldots, M$, of equal step size $h=T / M$, by using the nodes $x^{m}=m h$. Firstly, we apply the RKHS method to the following IVP: $u_{1}^{\prime \prime}(x)=f\left(x, u_{1}(x), u_{1}^{\prime}(x)\right), u_{1}(a)=\alpha, u_{1}^{\prime}(a)=\beta$, $x \in\left[0, x^{1}\right]$, to obtain the approximate solution $u_{1 n}(x)=$ $\sum_{i=1}^{n} A_{1 i} \bar{\psi}_{i}(x), x \in\left[0, x^{1}\right]$. For $m \geq 2$ and at each subinterval $\left[x^{m-1}, x^{m}\right]$, we will use the initial conditions $u_{m}\left(x^{m-1}\right)=$ $u_{(m-1) n}\left(x^{m-1}\right)=\alpha_{m}$ and $u_{m}^{\prime}\left(x^{m-1}\right)=u_{(m-1) n}^{\prime}\left(x^{m-1}\right)=\beta_{m}$ and then apply the RKHS method over the interval $\left[x^{m-1}, x^{m}\right]$. The process is repeated and generates a sequence of approximate solutions $u_{m n}(x), m=0,1, \ldots, M$ as follows:

$$
u_{n}(x)= \begin{cases}u_{1 n}(x)=\sum_{i=1}^{n} \sum_{k=1}^{i} \beta_{i k} F\left(x_{k}, v\left(x_{k}\right), v^{\prime}\left(x_{k}\right)\right) \bar{\psi}_{i}(x)+\beta x+(\alpha-a \beta), & x \in\left[0, x^{1}\right], \\ u_{2 n}(x)=\sum_{i=1}^{n} \sum_{k=1}^{i} \beta_{i k} F\left(x_{k}, v\left(x_{k}\right), v^{\prime}\left(x_{k}\right)\right) \bar{\psi}_{i}(x)+\beta_{2} x+\left(\alpha_{2}-a \beta_{2}\right), & x \in\left[x^{1}, x^{2}\right], \\ \vdots & \\ u_{M n}(x)=\sum_{i=1}^{n} \sum_{k=1}^{i} \beta_{i k} F\left(x_{k}, v\left(x_{k}\right), v^{\prime}\left(x_{k}\right)\right) \bar{\psi}_{i}(x)+\beta_{M} x+\left(\alpha_{M}-a \beta_{M}\right), & x \in\left[x^{M-1}, x^{M}\right],\end{cases}
$$

where $x_{i}=x^{m-1}+\left(x^{m}-x^{m-1}\right)((i-1) /(n-1))$ and $x_{i} \in$ $\left[x^{m-1}, x^{m}\right]$.

The spaces $W_{2}^{3}[a, b]$ and $W_{2}^{1}[a, b]$ are complete Hilbert with some special properties. So, all the properties of the Hilbert space will hold. Further, these spaces possess some special and better properties which could make some problems be solved easier. For instance, many problems studied in
$L^{2}[a, b]$ space, which is a complete Hilbert space, require large amount of integral computations and such computations may be very difficult in some cases. Thus, the numerical integrals have to be calculated at the cost of losing some accuracy. However, the properties of $W_{2}^{3}[a, b]$ and $W_{2}^{1}[a, b]$ require no more integral computation for some functions, instead of computing some values of a function at some nodes. 


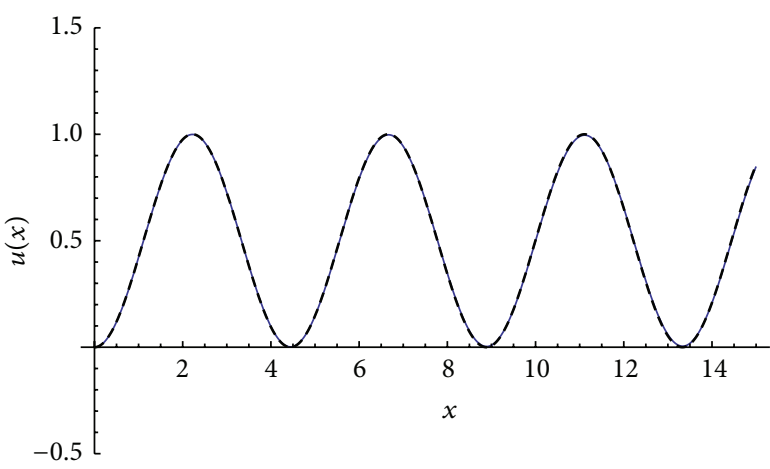

(a)

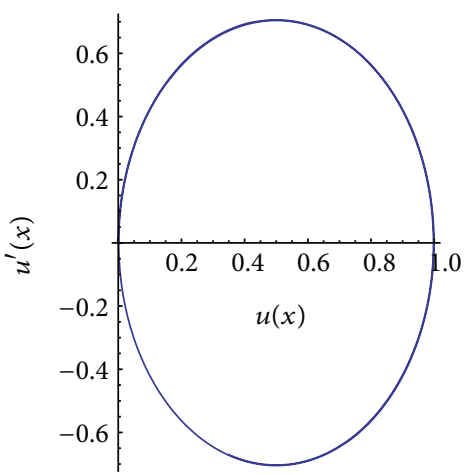

(b)

FIGURE 1: (a) Plots of displacement of $u$ versus time: solid line the multistep RKHS method and dashed line exact solution. (b) Phase plane diagram of Example 1.

In fact, this simplification of integral computation not only improves the computational speed, but also improves the computational accuracy.

\section{Numerical Examples and Graphical Results}

Numerical techniques are widely used by scientists and engineers to solve their problems. A major advantage for numerical techniques is that a numerical answer can be obtained even when a problem has no analytical solution. However, result from numerical analysis is an approximation, in general, which can be made as accurate as desired. Because a computer has a finite word length only a fixed number of digits are stored and used during computation. In order to demonstrate the applicability and effectiveness of the proposed algorithm, four examples will be solved numerically in this section.

Example 1. Consider the following linear oscillators equation: $u^{\prime \prime}(x)+2 u(x)-1=0$, subject to the initial conditions $u(0)=0, u^{\prime}(0)=0$. The exact solution is $u(x)=(1 / 2)(1-$ $\cos (\sqrt{2} x))$.

In this example, we apply the proposed algorithm on the interval $[0,15]$ and choose to divide the interval into subintervals with time step size $\Delta x=1$. In fact, assume that the interval $[0,15]$ is divided into 15 subintervals $\left[x^{m-1}, x^{m}\right]$, $m=1,2, \ldots, 15$, of equal step size $h=1 /(n-1)$. Anyhow, we apply RKHS method with $n=26$ in each IVP:

$$
\begin{array}{r}
L u_{i}(x)=1-2 u_{i}(x) ; \\
u_{i}\left(x_{i-1}\right)=u_{i-1}\left(x_{i-1}\right), \\
u_{i}^{\prime}\left(x_{i-1}\right)=u_{i-1}^{\prime}\left(x_{i-1}\right), \\
x \in\left[x_{i-1}, x_{i}\right] .
\end{array}
$$

The numerical results at some selected points in $[0,15]$ are given in Table 1, while, on the other aspect as well, Figure 1 shows that the results of our computations are in excellent agreement with the exact solution.
TABLE 1: Numerical results for Example 1.

\begin{tabular}{cccc}
\hline$x$ & Exact solution & Approximate solution & Absolute error \\
\hline 1 & 0.422028152617 & 0.422025539209 & $2.613408511 \times 10^{-6}$ \\
2 & 0.975681564063 & 0.975679846174 & $1.717889282 \times 10^{-6}$ \\
3 & 0.726330928646 & 0.726337897921 & $6.969275154 \times 10^{-6}$ \\
5 & 0.147326046846 & 0.147316914492 & $9.132353861 \times 10^{-6}$ \\
7 & 0.944711061916 & 0.944719149810 & $8.087893972 \times 10^{-6}$ \\
9 & 0.006510539200 & 0.006507180434 & $3.358766650 \times 10^{-6}$ \\
11 & 0.994264292331 & 0.994259400506 & $4.891824396 \times 10^{-6}$ \\
12 & 0.651670468807 & 0.651700265815 & $2.979700811 \times 10^{-5}$ \\
14 & 0.208928285637 & 0.208898850504 & $2.943513285 \times 10^{-5}$ \\
15 & 0.856178588587 & 0.856150469874 & $2.811871259 \times 10^{-5}$ \\
\hline
\end{tabular}

It is observed that the increase in the number of node results in a reduction in the absolute error and correspondingly an improvement in the accuracy of the obtained solution. This goes in agreement with the known fact; the error is monotone decreasing, where more accurate solutions are achieved using an increase in the number of nodes.

Example 2. Consider the following nonlinear oscillators equation: $u^{\prime \prime}(x)+u(x)+0.1 u^{2}(x) u^{\prime}(x)=0$, subject to the initial conditions $u(0)=1, u^{\prime}(0)=0$.

In this example, we apply the proposed algorithm on the interval $[0,150]$ and choose to divide the interval $[0,150]$ to subintervals with time step size $\Delta x=1$. Similarly, assume that the interval $[0,150]$ is divided into 150 subintervals $\left[x^{m-1}, x^{m}\right], m=1,2, \ldots, 150$, of equal step size $h=1 /(n-1)$. Anyhow, we apply RKHS method with $n=26$ in each IVP:

$$
\begin{aligned}
L u_{i}(x)=-u_{i}(x)-0.1 u_{i}^{2}(x) u_{i}^{\prime}(x) ; & \\
u_{i}\left(x_{i-1}\right)=u_{i-1}\left(x_{i-1}\right), \quad u_{i}^{\prime}\left(x_{i-1}\right) & =u_{i-1}^{\prime}\left(x_{i-1}\right), \\
x & \in\left[x_{i-1}, x_{i}\right] .
\end{aligned}
$$

Figure 2(a) shows that the results of our computations are in excellent agreement with the results obtained by 


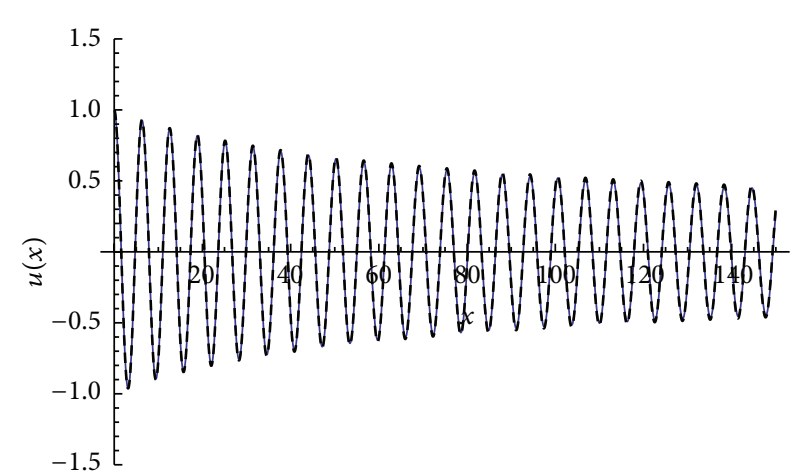

(a)

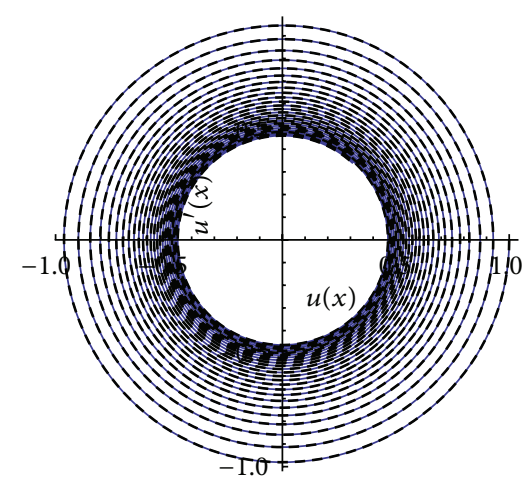

(b)

FIgURE 2: (a) Plots of displacement of $u$ versus time. (b) Phase plane diagram of Example 2: solid line the multistep RKHS method and dotted line RK method.

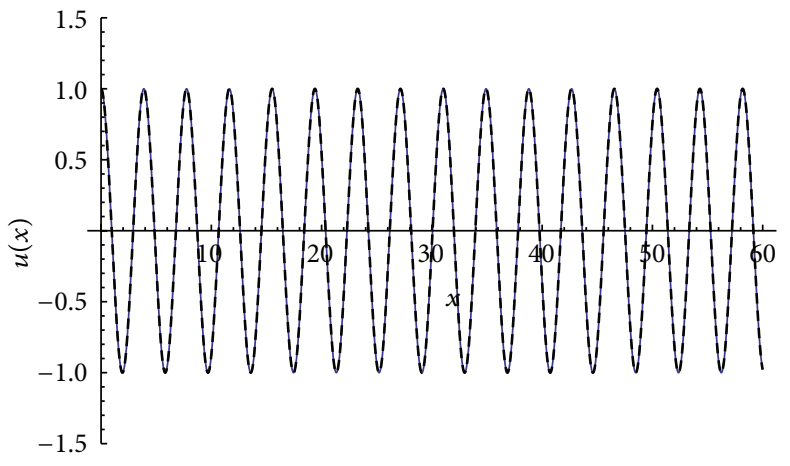

(a)

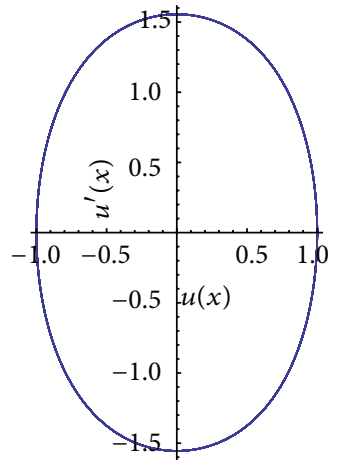

(b)

Figure 3: (a) Plots of displacement of $u$ versus time. (b) Phase plane diagram of Example 3: solid line the multistep RKHS method and dashed line RK method.

the numerical solution of [11] using multistep differential transform method. In Figure 2(b) we give a comparison between the multistep RKHS method and RK method for the problem.

This procedure can be repeated till the arbitrary order coefficients of the multistep RKHS solution are obtained. Moreover, higher accuracy can be achieved by evaluating more components of the solution.

Example 3. Consider the following nonlinear oscillators equation: $u^{\prime \prime}(x)+u(x)+u^{3}(x)+\sin (u(x))=0$, subject to the initial conditions $u(0)=1, u^{\prime}(0)=0$.

In this example, we apply the proposed algorithm on the interval $[0,60]$ and choose to divide the interval $[0,60]$ to subintervals with time step size $\Delta x=1$. In fact, assume that the interval $[0,60]$ is divided into 60 subintervals $\left[x^{m-1}, x^{m}\right]$, $m=1,2, \ldots, 60$, of equal step size $h=1 /(n-1)$. Anyhow, we apply RKHS method with $n=26$ in each IVP:

$$
\begin{aligned}
L u_{i}(x)=-u_{i}(x)-u_{i}^{3}(x)-\sin \left(u_{i}(x)\right) & ; \\
u_{i}\left(x_{i-1}\right)=u_{i-1}\left(x_{i-1}\right), \quad u_{i}^{\prime}\left(x_{i-1}\right) & =u_{i-1}^{\prime}\left(x_{i-1}\right), \\
x & \in\left[x_{i-1}, x_{i}\right] .
\end{aligned}
$$

As in the last example, Figure 3(a) shows that the results of our computations are in excellent agreement with the results obtained by the numerical solution of [11] using multistep differential transform method. On the other hand, in Figure 3(b), we give a comparison between the multistep RKHS method and RK method for the problem.

Example 4. Consider the following nonlinear oscillators equation: $u^{\prime \prime}(x)+u_{i}^{3}(x) /\left(1+u_{i}^{2}(x)\right)=0$, subject to the initial conditions $u(0)=0, u^{\prime}(0)=0.3$.

In this example, we apply the proposed algorithm on the interval $[0,100]$ and choose to divide the interval $[0,100]$ to subintervals with time step size $\Delta x=1$. Similarly, assume that the interval $[0,100]$ is divided into 100 subintervals $\left[x^{m-1}, x^{m}\right], m=1,2, \ldots, 100$, of equal step size $h=1 /(n-1)$. Anyhow, we apply RKHS method with $n=26$ in each IVP:

$$
\begin{array}{r}
L u_{i}(x)=\frac{-u_{i}^{3}(x)}{\left(1+u_{i}^{2}(x)\right)} ; \\
u_{i}\left(x_{i-1}\right)=u_{i-1}\left(x_{i-1}\right), \\
u_{i}^{\prime}\left(x_{i-1}\right)=u_{i-1}^{\prime}\left(x_{i-1}\right), \\
x \in\left[x_{i-1}, x_{i}\right] .
\end{array}
$$




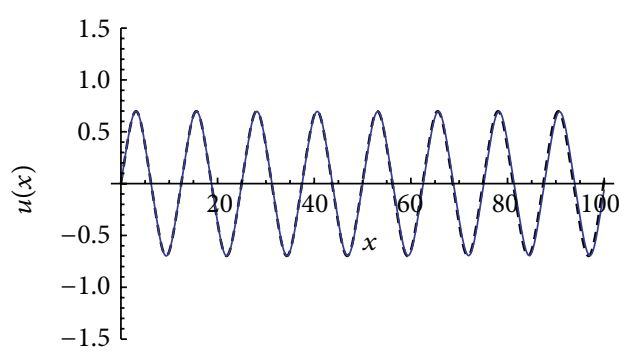

(a)

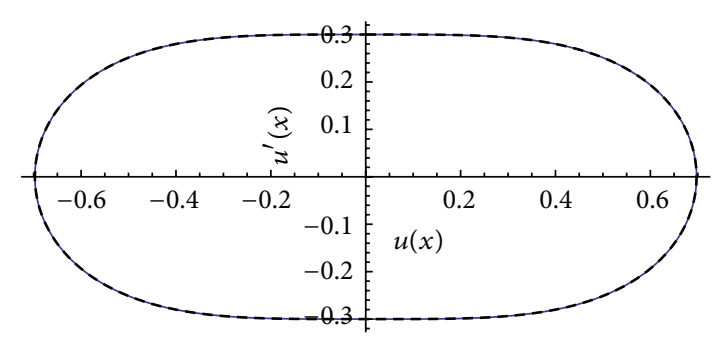

(b)

FIgURE 4: (a) Plots of displacement of $u$ versus time. (b) Phase plane diagram of Example 4: solid line the multistep RKHS method and dashed line RK method.

As a result, Figure 4(a) shows that the results of our computations are in excellent agreement with the results obtained by the numerical solution of [11] using multistep differential transform method. Anyhow, in Figure 4(b), we give a comparison between the multistep RKHS method and RK method for the problem.

\section{Conclusions}

In this study, a new algorithm is proposed for finding a numerical solution of linear and nonlinear oscillators, namely, multistep reproducing kernel Hilbert space method. The main characteristic feature of the multistep RKHS method is that the global approximation can be established on the whole solution domain, in contrast with other numerical methods like one step and multistep methods, and the convergence is uniform. Indeed, the present method is accurate, needs less effort to achieve the results, and is especially developed for nonlinear case. On the other aspect as well, the derivatives of the approximate solutions are also uniformly convergent. Comparison results between multistep RKHS method solution and RK method are discussed; the results show that this method is accurate for solving this kind of equations.

\section{Conflict of Interests}

The authors declare that there is no conflict of interests regarding the publication of this paper.

\section{References}

[1] L. Dai and R. N. Jazar, Nonlinear Approaches in Engineering Applications, Springer, New York, NY, USA, 2012.

[2] S. Strogatz, Nonlinear Dynamics and Chaos: With Applications to Physics, Biology, Chemistry, and Engineering, Perseus Books, Cambridge, Mass, USA, 1994.

[3] A. H. Nayfeh, Perturbation Methods, John Wiley \& Sons, New York, NY, USA, 1973.

[4] A. H. Nayfeh and D. T. Mook, Nonlinear Oscillations, John Wiley \& Sons, New York, NY, USA, 1979.

[5] M. Ghadimi and H. D. Kaliji, "Application of the harmonic balance method on nonlinear equations," World Applied Sciences Journal, vol. 22, no. 4, pp. 532-537, 2013.
[6] F. Lakrad and M. Belhaq, "Periodic solutions of strongly nonlinear oscillators by the multiple scales method," Journal of Sound and Vibration, vol. 258, no. 4, pp. 677-700, 2002.

[7] N. Movasaghi and H. Kheiri, Application of Adomian Decomposition Method to Nonlinear Oscillators, World Scientific and Engineering Academy and Society, 2009.

[8] S. Momani, G. H. Erjaee, and M. H. Alnasr, "The modified homotopy perturbation method for solving strongly nonlinear oscillators," Computers \& Mathematics with Applications, vol. 58, no. 11-12, pp. 2209-2220, 2009.

[9] M. Zurigat, S. Momani, and A. Alawneh, "The multi-step homotopy analysis method: a powerful scheme for handling non-linear oscillators," Iranian Journal of Science \& Technology, vol. 37, no. 3, pp. 421-429, 2013.

[10] O. Abu-Arqub, A. El-Ajou, S. Momani, and N. Shawagfeh, "Analytical solutions of fuzzy initial value problems by HAM," Applied Mathematics \& Information Sciences, vol. 7, no. 5, pp. 1903-1919, 2013.

[11] V. S. Ertürk, Z. M. Odibat, and S. Momani, “The multi-step differential transform method and its application to determine the solutions of non-linear oscillators," Advances in Applied Mathematics and Mechanics, vol. 4, no. 4, pp. 422-438, 2012.

[12] S. Momani, "Analytical approximate solutions of nonlinear oscillators by the modified decomposition method," International Journal of Modern Physics C, vol. 15, no. 7, pp. 967-979, 2004.

[13] J. Langari and M. Akbarzade, “The he's amplitude-frequency formulation for solving strongly nonlinear oscillators differential equations," Advanced Studies in Theoretical Physics, vol. 5, no. 5-8, pp. 343-348, 2011.

[14] J. I. Ramos, "An artificial parameter-decomposition method for nonlinear oscillators: Applications to oscillators with odd nonlinearities," Journal of Sound and Vibration, vol. 307, no. 1-2, pp. 312-329, 2007.

[15] A. Ebaid, S. Momani, S. H. Chang, and M. Aljoufi, "On the periodic solutions of the nonlinear oscillators," Journal of Vibroengineering, vol. 16, no. 1, pp. 1-15, 2014.

[16] B. Soltanalizadeh, "Differential transformation method for solving one-space-dimensional telegraph equation," Computational \& Applied Mathematics, vol. 30, no. 3, pp. 639-653, 2011.

[17] B. Soltanalizadeh and A. Hajihosseini, "A new efficient technique for the numerical computation of the linear Boussinesq equation," Wulfenia Journal, vol. 19, no. 8, pp. 123-137, 2012.

[18] A. Berlinet and C. Thomas-Agnan, Reproducing Kernel Hilbert Spaces in Probability and Statistics, Kluwer Academic Publishers, Boston, Mass, USA, 2004. 
[19] M. Cui and Y. Lin, Nonlinear Numerical Analysis in the Reproducing Kernel Space, Nova Science, New York, NY, USA, 2009.

[20] A. Daniel, Reproducing Kernel Spaces and Applications, Springer, 2003.

[21] F. Geng and M. Cui, "Solving a nonlinear system of second order boundary value problems," Journal of Mathematical Analysis and Applications, vol. 327, no. 2, pp. 1167-1181, 2007.

[22] Y. Li, F. Geng, and M. Cui, "The analytical solution of a system of nonlinear differential equations," International Journal of Mathematical Analysis, vol. 1, no. 9-12, pp. 451-462, 2007.

[23] M. Cui and F. Geng, "Solving singular two-point boundary value problem in reproducing kernel space," Journal of Computational and Applied Mathematics, vol. 205, no. 1, pp. 6-15, 2007.

[24] E. Gao, S. Song, and X. Zhang, "Solving singular secondorderinitial/boundary value problems in reproducing Kernel Hilbert space," Boundary Value Problems, vol. 2012, article 3, 2012.

[25] N. Shawagfeh, O. Abu Arqub, and S. Momani, "Analytical solution of nonlinear second-order periodic boundary value problem using reproducing kernel method," Journal of Computational Analysis and Applications, vol. 16, no. 4, pp. 750-762, 2014.

[26] O. Abu Arqub, M. Al-Smadi, and S. Momani, "Application of reproducing kernel method for solving nonlinear FredholmVolterra integrodifferential equations," Abstract and Applied Analysis, vol. 2012, Article ID 839836, 16 pages, 2012.

[27] O. A. Arqub, M. Al-Smadi, and N. Shawagfeh, "Solving Fredholm integro-differential equations using reproducing kernel Hilbert space method," Applied Mathematics and Computation, vol. 219, no. 17, pp. 8938-8948, 2013.

[28] M. Al-Smadi, O. Abu Arqub, and S. Momani, "A computational method for two-point boundary value problems of fourth-order mixed integrodifferential equations," Mathematical Problems in Engineering, vol. 2013, Article ID 832074, 10 pages, 2013.

[29] M. Al-Smadi, O. Abu Arqub, and A. El-Ajuo, "A numerical method for solving systems of first-order periodic boundary value problems," Journal of Applied Mathematics, vol. 2014, Article ID 135465, 10 pages, 2014.

[30] O. Abu Arqub, "An iterative method for solving fourth-order boundary value problems of mixed type integro-differential equations," Journal of Computational Analysis and Applications. In press.

[31] S. Bushnaq, S. Momani, and Y. Zhou, "A reproducing kernel Hilbert space method for solving integro-differential equations of fractional order," Journal of Optimization Theory and Applications, vol. 156, no. 1, pp. 96-105, 2013.

[32] S. Bushnaq, B. Maayah, S. Momani, and A. Alsaidi, "A reproducing Kernel Hilbert space method for solving systems of fractional integro-differential equations," Abstract and Applied Analysis, vol. 2014, Article ID 103016, 6 pages, 2014. 


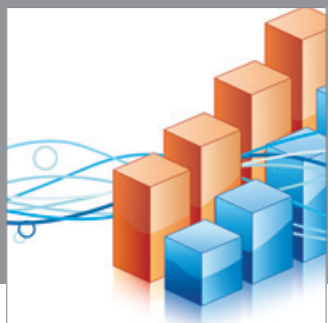

Advances in

Operations Research

mansans

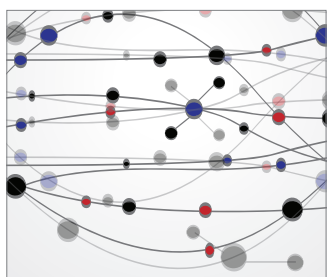

The Scientific World Journal
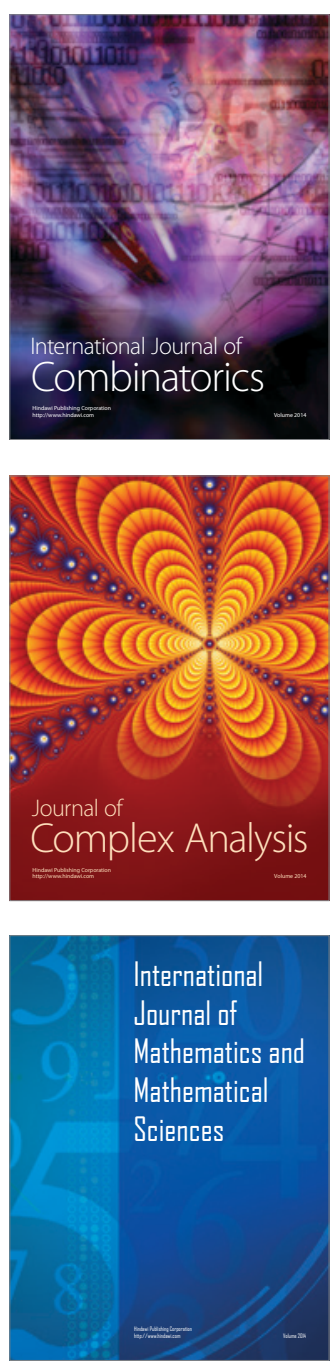
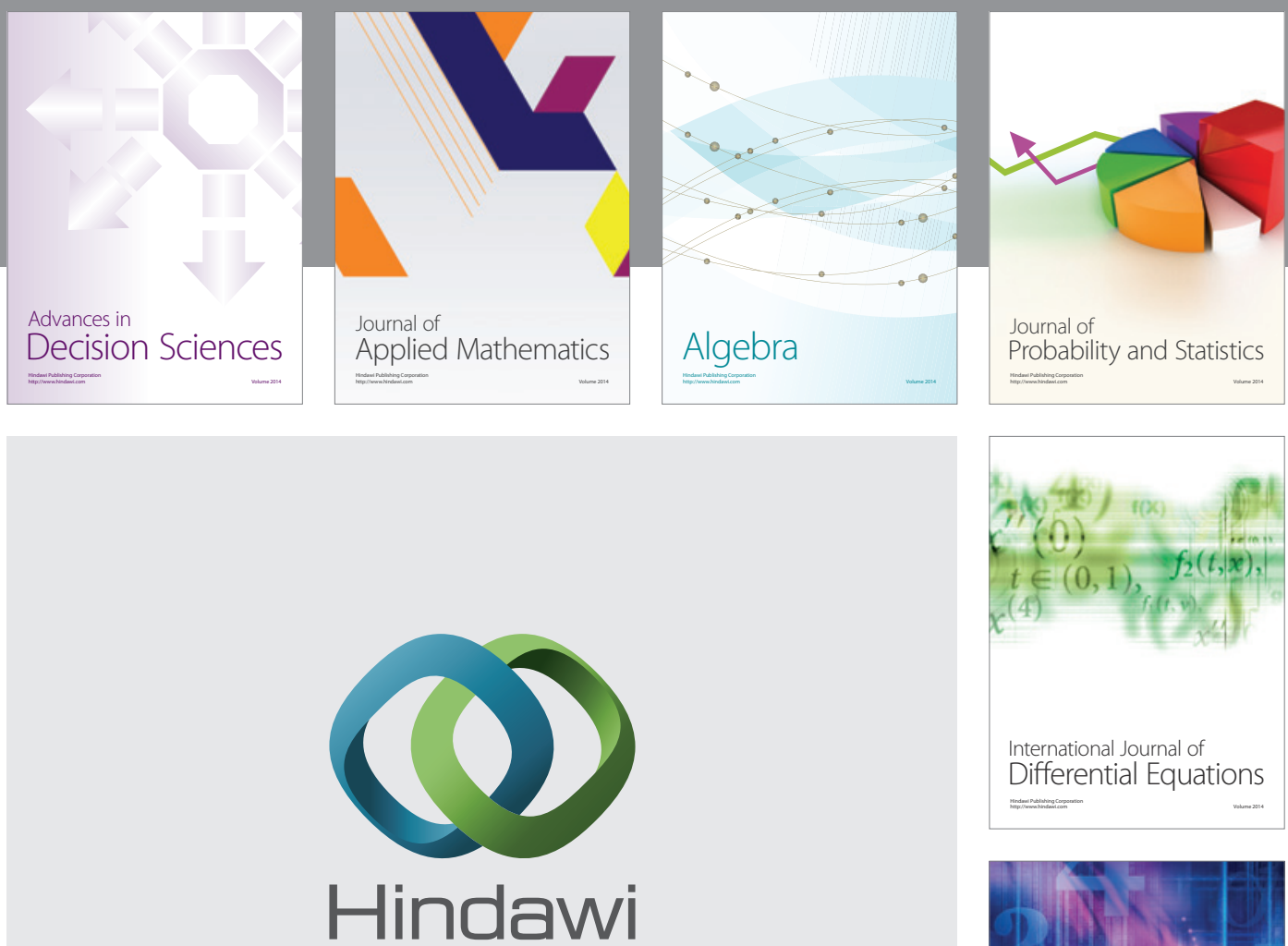

Submit your manuscripts at http://www.hindawi.com
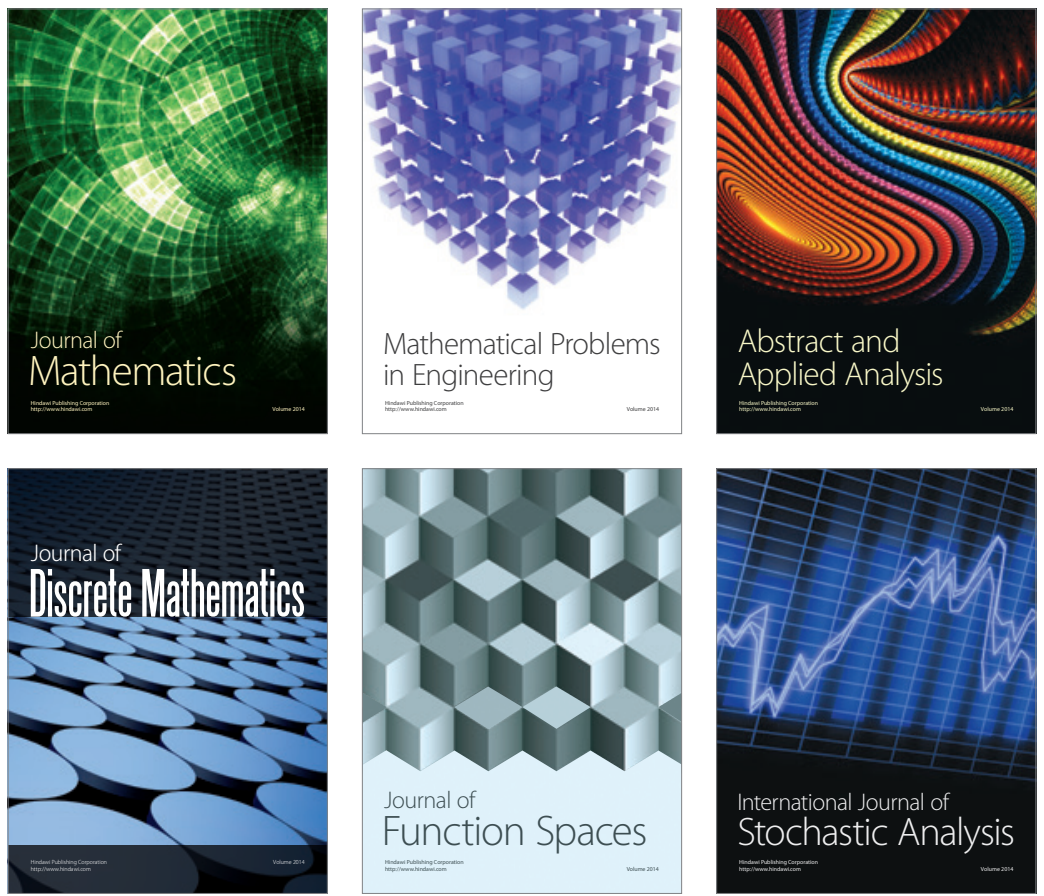

Journal of

Function Spaces

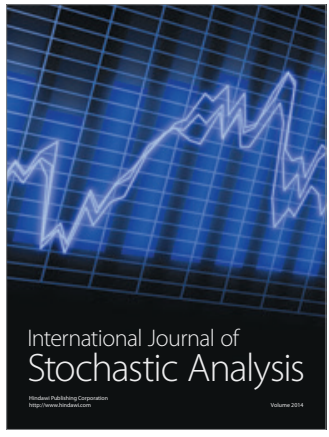

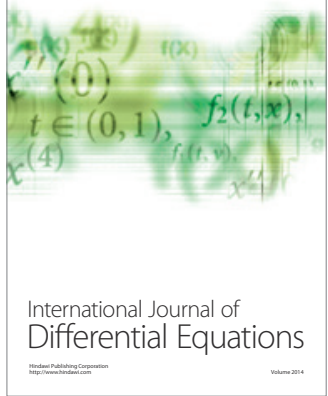
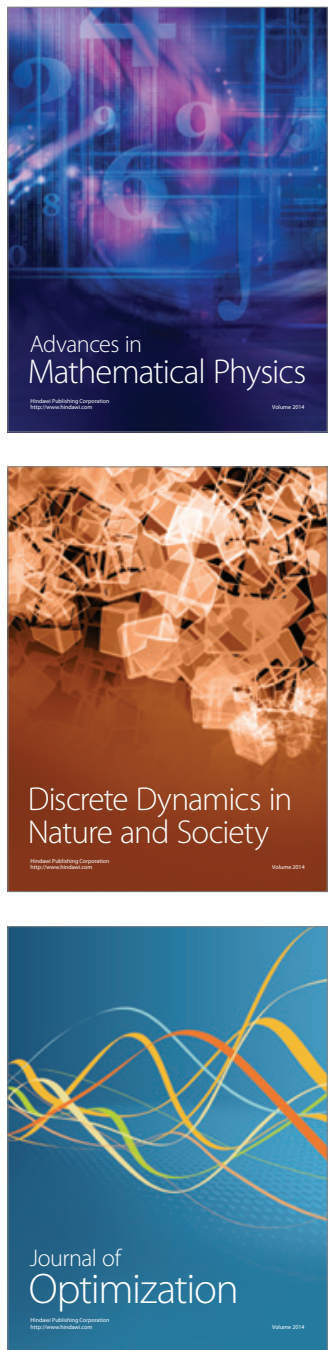